\title{
Mechanisms of Hippocampal Long-Term Depression Are Required for Memory Enhancement by Novelty Exploration
}

\author{
Zhifang Dong, ${ }^{1,2,3}$ Bo Gong, ${ }^{4}$ Hongjie Li, ${ }^{1,2,3}$ Yanrui Bai, ${ }^{1,2,3}$ Xiaoyan Wu, ${ }^{1,2,3}$ Yan Huang, ${ }^{1,2,3}$ Wenting He, ${ }^{1,2,3}$ Tingyu Li, ${ }^{1,2,3}$ \\ and Yu Tian Wang ${ }^{1,2,3,4}$ \\ ${ }^{1}$ Ministry of Education Key Laboratory of Child Development and Disorders, ${ }^{2}$ Chongqing Key Laboratory of Translational Medical Research in Cognitive \\ Development and Learning and Memory Disorders, ${ }^{3}$ Chongqing International Science and Technology Cooperation Center for Child Development and \\ Disorders, Children's Hospital of Chongqing Medical University, Chongqing 400014, People’s Republic of China, and ${ }^{4}$ Brain Research Centre, University of \\ British Columbia, Vancouver, British Columbia V6T 2B5, Canada
}

It is well known that novel environments can enhance learning and memory. However, the underlying mechanisms remain poorly understood. Here, we report that, in freely moving rats, novelty exploration facilitates the production of hippocampal CA1 long-term depression (LTD), a well characterized form of synaptic plasticity believed to be a cellular substrate of spatial learning, and thereby converts short-term memory (STM) into long-term memory (LTM) in an inhibitory avoidance learning procedure. Blocking the induction or the expression of CA1 LTD with two mechanistically and structurally distinct inhibitors prevents not only novelty acquisition but also the novelty exploration-promoted conversion of STM into LTM. Moreover, production of LTD with a strong electrical stimulation induction protocol or facilitation of hippocampal LTD by pharmacological inhibition of glutamate transporter activity mimics the behavioral effects of novelty exploration, sufficiently promoting the conversion of STM into LTM. Together, our findings suggest that induction of LTD may play an essential role not only in novelty acquisition but also in novelty-mediated memory enhancement.

\section{Introduction}

It is well documented that exposures to a novel environment can enhance memory (Moncada and Viola, 2007; Ballarini et al., 2009). Although the detailed underlying mechanisms remain poorly characterized, the hippocampus has been implicated in both detection of novelty (Zhu et al., 1997; Honey et al., 1998; Manahan-Vaughan and Braunewell, 1999) and memory formation (Izquierdo and Medina, 1997; Riedel et al., 1999; Szapiro et al., 2000; Lemon and Manahan-Vaughan, 2006). Thus, the hippocampus may be one of the critical brain areas for memory enhancement by exposure to novel environment (Jenkins et al., 2004). Activity-dependent synaptic plasticity at hippocampal glutamatergic synapses, particularly long-term potentiation (LTP) and long-term depression (LTD) at the CA3-CA1 synapse, has been proposed as the cellular substrate of information pro-

Received Feb. 28, 2012; revised July 4, 2012; accepted July 11, 2012.

Author contributions: Z.D., T.L., and Y.T.W. designed research; Z.D., B.G., H.L., Y.B., X.W., Y.H., and W.H. performed research; Y.T.W. contributed unpublished reagents/analytic tools; Z.D., B.G., H.L., Y.B., and X.W. analyzed data; Z.D., B.G., and Y.T.W. wrote the paper.

This work was supported by the Canadian Institutes of Health Research (Y.T.W.), 973 Program of the Ministry of Science and Technology of China Grant 2012CB517903, National Natural Science Foundation of China Grant 31040085, Chongqing International Science and Technology Cooperation Foundation cstc201110003, and Canadian Institutes of Health Research Fellowship (Z.D.), and National Natural Science Foundation of China Grants 30830106 and 30972461 (T.L.). B.G. was supported by a Doctoral Award from the Canadian Institutes of Health Research. We thank Dr. Loren Oschipok for editorial assistance.

The authors declare no competing financial interests.

Correspondence should be addressed to either of the following: Dr. Yu Tian Wang, F117-2211 Wesbrook Mall, Vancouver, BC V6T 2B5, Canada, E-mail: ytwang@brain.ubc.ca; or Dr. Zhifang Dong, 136 Zhongshan Er Road, Yuzhong District, Chongqing 400014, People's Republic of China. E-mail: zhifang.dong@gmail.com.

DOI:10.1523/JNEUROSCI.0984-12.2012

Copyright $\odot 2012$ the authors $\quad 0270-6474 / 12 / 3211980-11 \$ 15.00 / 0$ cessing and memory formation (Bliss and Collingridge, 1993; Malenka and Nicoll, 1999; Martin et al., 2000). While accumulating evidence supports a critical role of hippocampal LTP and LTD in spatial learning (O'Keefe and Nadel, 1978; Morris et al., 1982; Whitlock et al., 2006; Ge et al., 2010), it is currently unknown whether hippocampal LTP or LTD are critical to memory enhancement produced by novelty exploration. However, several recent studies have reported that hippocampal LTD is facilitated by novelty exposure (Manahan-Vaughan and Braunewell, 1999), and possibly important for novelty acquisition (Lemon and Manahan-Vaughan, 2006). Since we have recently found that induction of hippocampal CA1 LTD promotes the consolidation of spatial learning in freely moving rats (Ge et al., 2010), we hypothesize that the induction of hippocampal LTD, promoted by novel exploration, may be a critical mechanism for novelty exposure-induced memory enhancement. In the present study, we investigated this hypothesis using a combination of electrophysiological and behavioral assessments in freely moving rats.

\section{Materials and Methods}

Animals

Adult male Sprague Dawley rats (350 - 400 g; obtained from University of British Columbia Animal Care Centre) were housed in plastic cages in a temperature-controlled $\left(21^{\circ} \mathrm{C}\right)$ colony room on a $12 \mathrm{~h}$ light/dark cycle. Food and water were available ad libitum. All experiments were performed in accordance with the Canadian Council on Animal Care and were approved by the University of British Columbia Animal Care Committee. All efforts were made to minimize animal discomfort and to reduce the number of animals used. 


\section{Drugs and treatment procedures}

The specific GluN2B antagonist $\left[R-\left(R^{*}, S^{*}\right)\right]-\alpha$-(4-hydroxyphenyl)- $\beta$ methyl-4-( phenylmethyl)-1-piperidinepropanol (Ro25-6981) was obtained from Sigma-Aldrich. DL-threo- $\beta$-Benzyloxyaspartate (DL-TBOA) and $(R S)$ - $\alpha$-methyl-4-carboxyphenylglycine (MCPG) were purchased from Tocris Bioscience. Tat-GluA2 ${ }_{3 Y}$ (YGRKKRRQRRR- ${ }_{869}$ YKEGYNVYG $_{877}$ ) or scrambled Tat-GluA2 ${ }_{3 Y}$ (YGRKKRRQRRR- ${ }_{869}$ VYKYGGYNE $_{877}$ ) peptides were synthesized by GL Biochem Ltd. MCPG (500 nmol in $5 \mu \mathrm{l}$ for intracerebroventricular infusion), Ro25-6981 (6.0 mg/kg for intraperitoneal or $0.5 \mathrm{nmol} / \mu \mathrm{l}$ per side for intrahippocampal infusion), DL-TBOA (1 $\mathrm{nmol} / \mu \mathrm{l}$ per side for intrahippocampal infusion or $5 \mathrm{nmol}$ in $5 \mu \mathrm{l}$ for intracerebroventricular infusion) and Tat-peptides $(3.0 \mu \mathrm{mol} / \mathrm{kg}$ for intraperitoneal injection or $100 \mathrm{pmol} / \mu \mathrm{l}$ per side for intrahippocampal infusion) were dissolved in $0.9 \%$ sterile saline at required concentrations.

\section{Bilateral hippocampal microinjection}

Surgery. Rats were chronically implanted with cannulas above dorsal hippocampi as previously described (Dong et al., 2006; Ge et al., 2010). Briefly, rats were anesthetized with sodium pentobarbital $(60 \mathrm{mg} / \mathrm{kg}$, i.p.). Atropine ( $0.4 \mathrm{mg} / \mathrm{kg}$, i.p.) was also given to help relieve respiratory congestion. Scalp skin was shaved by a clipper and disinfected using iodine before the rat was mounted on a stereotaxic instrument. After opening the scalp skin and exposing the skull, two 22 gauge stainless-steel guide cannulas (10 mm; Plastics One) were implanted above the dorsal hippocampi (3.8 $\mathrm{mm}$ posterior to bregma, $2.5 \mathrm{~mm}$ lateral to the midline, and $2.5 \mathrm{~mm}$ below the surface of the dura) and fixed to the skull with four jeweler's screws and dental cement. Sterile dummy cannula (30 gauge stainless-steel rod; $10 \mathrm{~mm}$ in length; Plastics One) were inserted into guide cannula to avoid bacterial infection and CSF leakage through the cannula. All rats were allowed to recover for 7-10 d before experiments.

Habituation. On the day before experiments, the animals were placed in the experiment room and given a sham intrahippocampal injection to become acclimatized to the injection procedure. Dummy cannulas were removed and the rats were placed into a Plexiglas injection box $(25 \times$ $45 \times 25 \mathrm{~cm}$; same as home cage) with 30 gauge injection cannulas in their guide cannulas. Injection cannulas (11 mm; Plastics One) were connected to a microsyringe pump (Harvard Apparatus) by PE-50 tubing, which are $1 \mathrm{~mm}$ beyond the tip of the guide cannulas.

Intrahippocampal microinjection. Drugs were injected with $10 \mu \mathrm{l} \mathrm{Ham-}$ ilton syringes and a microsyringe pump at $0.5 \mu \mathrm{l} / \mathrm{min}$ for $2 \mathrm{~min}$. After injection, the injection cannulas were left in place for an additional minute to allow the diffusion of the drug away from the cannula tips. The rats were then removed from the injection box, their dummy cannulas were replaced, and they were placed back in their home cages. The cannula placement was verified by histological examination of the brain after methylene blue injection ( $1 \mu \mathrm{l}$ per side), and only data obtained from rats with correctly inserted cannulas were included in statistical analysis ( $\sim 10 \%$ animals were excluded from the experiment due to nonfunctional cannulas or postoperative complications).

\section{Novelty exploration}

The novelty exploration apparatus is a black Plexiglas box $(40 \times 40 \times 60$ $\mathrm{cm})$. The floor of the box was covered with sawdust. Each corner of the box contained a different object ( $5 \mathrm{~cm}$ from the walls). The objects differed from each other in appearance and size. The box was open at the top to let animals locate the position of each object from surrounding environment that served as visual cues. Three trials, each lasting for $15 \mathrm{~min}$, were conducted with $24 \mathrm{~h}$ intertrial intervals. One hour before the first trial, 12 rats received saline, 12 rats received Ro25-6981, 9 rats received scrambled Tat-GluA2 $2_{3 Y}$ (sTatGluA2 $2_{3 Y}$ ) peptide, and 12 rats received Tat-GluA2 $2_{3 Y}$ peptide injection. The experimenter recorded the occurrence of head dips to the objects. No injections were given on subsequent exposures to the objects.

\section{Inhibitory avoidance task}

The inhibitory avoidance (IA) apparatus is a two-chambered Perspex box consisting of a lighted safe compartment and a dark shock compartment separated by a trap door $(35 \times 30 \times 35 \mathrm{~cm}$ for each compartment; Coulbourn Instruments). One day before training, rats were allowed to freely explore both chambers for $3 \mathrm{~min}$. On the training day, rats received 15 min exploration of novel objects when necessary and were then re- moved from the exploration box and placed back in the home cage for $1 \mathrm{~h}$ before they were subjected to IA training. During training, rats were placed in the safe compartment facing a corner opposite the door. Ten seconds later, the trap door was opened to allow animals to enter the dark compartment. After the animals entered the dark compartment, the door was closed and they received a weak (wIA) $(0.2 \mathrm{~mA}, 2 \mathrm{~s}$ ) or strong (sIA) $(0.4 \mathrm{~mA}, 2 \mathrm{~s})$ footshock via electrified steel rods on the box floor. The animals were given $15 \mathrm{~s}$ to recover in the dark compartment before being returned to their home cages. The animals were submitted to a test session to measure short-term memory (STM) (1 h after training) or long-term memory (LTM) (24 h after training). Memory was assessed by placing trained animals back in the lighted compartment of the box and measuring latencies for the animals to reenter the dark compartment. Reentry was counted when all of the animal's four paws were back in the dark compartment of the box. No footshock was administered during retention assays, and measurements were terminated at a ceiling test latency of $540 \mathrm{~s}$.

\section{Electrophysiological recordings}

Surgery and electrodes implantation. Rats were chronically implanted with electrodes as described previously (Xu et al., 1997; Dong et al., 2008; Ge et al., 2010). Briefly, subjects were anesthetized deeply with sodium pentobarbital (60 mg/kg, i.p.) and pretreated with atropine $(0.4 \mathrm{mg} / \mathrm{kg}$, i.p.) to prevent excessive salivation. The core temperature of anesthetized rats was maintained at $36.5 \pm 0.5^{\circ} \mathrm{C}$. Three stainless-steel bone anchor screws (Stoelting Co.) were inserted into the skull through drilled holes without piercing the dura. One screw served as a ground electrode $(7 \mathrm{~mm}$ posterior to bregma and $5 \mathrm{~mm}$ left of the midline), another screw acted as an anchor (opposite the ground screw, $7 \mathrm{~mm}$ posterior to bregma and 5 $\mathrm{mm}$ right of the midline), and the third screw served as a reference electrode ( $8 \mathrm{~mm}$ anterior to bregma and $1 \mathrm{~mm}$ left of the midline). Stimulating electrode was made by gluing together a pair of twisted Teflon-coated $90 \%$ platinum $/ 10 \%$ iridium wires (50 $\mu \mathrm{m}$ inner diameter, $100 \mu \mathrm{m}$ outer diameter; A-M Systems). Recording electrode was a single Teflon-coated platinum iridium wire (75 $\mu \mathrm{m}$ inner diameter; $140 \mu \mathrm{m}$ outer diameter; A-M Systems). The recording electrode was lowered into the CA1 pyramidal cell dendritic layer $(3.8 \mathrm{~mm}$ posterior to bregma, $2.8 \mathrm{~mm}$ right of the midline, and $\sim 2.5 \mathrm{~mm}$ below the surface of the dura), and the stimulating electrode was placed in the Schaffer collateral/commissural pathways of the dorsal hippocampus ( $4.5 \mathrm{~mm}$ posterior to bregma, $3.8 \mathrm{~mm}$ right of the midline, and $\sim 2.8 \mathrm{~mm}$ below the dura) via holes drilled through the skull. The electrode socket assembly was fixed onto the skull with dental cement. The correct placement of the electrodes in the CA1 region of the dorsal hippocampus was verified by electrophysiological criteria (Leung, 1979) and postmortem examination.

Electrophysiological recordings. All rats were allowed to recover for at least $7 \mathrm{~d}$ before freely moving recordings were performed. During this recovery period, rats were allowed to acclimatize the recording chamber $(40 \times 40 \times$ $60 \mathrm{~cm}$ ), which was made of black Plexiglas and open at the top, for at least $1 \mathrm{~h}$ each day. To allow rats to move around freely in the chamber during recording, implanted electrodes were connected by a flexible cable and a swivel commutator (Crist Instrument) to the stimulation and recording equipment. Field EPSPs (fEPSPs) were evoked by square-wave stimulations (pulse width, $0.12 \mathrm{~ms}$ ). Test fEPSPs were evoked at a frequency of $0.033 \mathrm{~Hz}$ and at a stimulus intensity adjusted to $\sim 50 \%$ of the maximal response size. After a 30 min stable baseline, LTD or LTP was induced. In the present experiment, two low-frequency stimulation (LFS) protocols were used to elicit LTD: (1) classical LFS (900 pulses at $1 \mathrm{~Hz}$ ) and (2) paired-burst stimulation (200 pairs of two-pulse bursts at one pair per second, with an interval of $2.5 \mathrm{~ms}$ between pulses and $10 \mathrm{~ms}$ between bursts). Two high-frequency stimulation (HFS) protocols were used to induce LTP. To induce an unsaturated form of LTP that contains only early-phase LTP (eLTP) (lasts for $<1.5 \mathrm{~h}$ ), a weak HFS (wHFS) that contains two trains of 30 pulses at $100 \mathrm{~Hz}$ were given with an intertrain interval of $5 \mathrm{~min}$. To induce the stable form of LTP that contains both early and late phases of LTP (over $24 \mathrm{~h}$ ), a strong HFS (sHFS) that contains four trains of 30 pulses at $100 \mathrm{~Hz}$, with an intertrain interval of 5 min, were given. During LTD or LTP induction in novel objects exploration and reexploration groups, rats were transferred from the baseline recording chamber (recording chamber) to another identical chamber (induction chamber) that contained four different objects in the corners. The objects 
differed from each other in appearance and size. The rats were immediately returned to recording chamber after HFS or LFS. In the control group, rats were transferred to induction chamber in the absence of objects during HFS or LFS delivery, and then were returned to the recording chamber.

\section{Statistical analysis}

Electrophysiological experiments. Field EPSPs evoked from 10 consecutive stimulations over 5 min were averaged to obtain a data point. All data were expressed as the average percentage change from baseline \pm SEM. LTD or LTP comparisons were made by using paired $t$ test compared with baseline. Between-groups comparisons were conducted by one-way ANOVA followed by Fisher's test. Significance level was set at $p<0.05$.

Novelty exploration. All data are expressed as mean \pm SEM. Statistical significance for exploring behavior (head-dipping) was analyzed with oneway ANOVA. Post hoc analysis was applied by using Fisher's test for different trials.

IA experiments. All data are presented as mean \pm SEM. Statistical significance for IA test was analyzed with one-way ANOVA. Post hoc analysis was applied by using Fisher's test for between-subjects and paired $t$ test for within-subjects comparisons.

\section{Results}

\section{Novelty exploration specifically facilitates the induction of hippocampal LTD}

fEPSPs evoked by the stimulation of the Schaffer collateral/commissural pathways of the dorsal hippocampus were recorded in the CA1 region of the hippocampus from freely moving rats (Fig. $1 A$ ). During the recordings, animals were allowed to explore new objects (exploration) or familiar objects (reexploration) (i.e., objects had been exposed to the animal for a 15 min 24 h earlier) for a period of $15 \mathrm{~min}$. We found that neither exploration nor reexploration significantly affected fEPSPs (control: $n=4,99.5 \pm 2.0 \%$, $p=0.446$ vs baseline; exploration: $n=4,99.4 \pm 1.1 \%, p=0.136$ vs baseline; reexploration: $n=4,99.9 \pm 2.8 \%, p=0.498$ vs baseline; Fig. $1 B$ ). We then examined whether novelty exploration can affect the production of hippocampal synaptic plasticity, LTP and LTD. Consistent with the previously reported difficulty in inducing hippocampal LTD in adult rats in vivo (Staubli and Scafidi, 1997; Xiong et al., 2004), we found that the standard LFS protocol (900 pulses at $1 \mathrm{~Hz}$ ) failed to induce LTD in the control group (control: $n=8,95.4 \pm 4.4 \%, p=0.232$ vs baseline; Fig. $1 C)$. However, when the rat was allowed to explore new objects while LFS was delivered, LFS reliably induced a LTD of fEPSPs over an observation period of $24 \mathrm{~h}$ (exploration: $n=7,71.1 \pm$ $2.8 \%, p<0.001$ vs baseline, $p=0.005$ vs control; Fig. $1 C$ ). To determine whether the facilitation of LTD is due to the novel nature of the objects, a group of animals was allowed a period of 15 min exposure to the novel objects $24 \mathrm{~h}$ before LTD induction before the animals were then reexposed to the same objects during LFS delivery period. As shown in Figure $1 C$, the reexposure to the same set of objects failed to facilitate the production of LTD by LFS (reexploration: $n=5,96.9 \pm 8.2 \%, p=0.349$ vs baseline, $p=0.974$ vs control; Fig. $1 C$ ). These results suggest that exploration of novel rather than familiar objects facilitates hippocampal CA1 LTD induction in freely moving animals. As previously reported (Ge et al., 2010), we found that a sHFS protocol (a four train of 30 pulses at $100 \mathrm{~Hz}$, with an intertrain interval of $5 \mathrm{~min}$ ) reliably produced a robust LTP of fEPSPs over the $24 \mathrm{~h}$ observation period in control animals (control: $n=7,128.8 \pm 3.8 \%, p<$ 0.001 vs baseline; Fig. $1 D$ ). Notably, neither novel object exploration nor reexploration significantly altered sHFS-induced LTP (exploration: $n=7,127.2 \pm 7.4 \%, p=0.006$ vs baseline, $p=$ 0.390 vs control; reexploration: $n=5,133.1 \pm 4.3 \%, p<0.001$ vs baseline, $p=0.342$ vs control; Fig. $1 D$ ). To exclude the possibility that the sHFS protocol induced saturated LTP that precludes the observation of any effect of novelty exploration on hippocampal LTP induction, we also examined whether the novel object exploration affects synaptic plasticity induced by a wHFS protocol (a two train of 30 pulses at $100 \mathrm{~Hz}$, with an intertrain interval of 5 min). Unlike the sHFS, wHFS could only produce an eLTP that lasted for $<1.5$ h (control: $n=4,97.7 \pm 4.8 \%, p=0.278$ vs baseline; Fig. $1 E$ ). We found that the novel exploration failed to alter this eLTP either (exploration: $n=4,100.2 \pm 3.5 \%, p=$ 0.295 vs baseline, $p=0.390$ vs control; Fig. $1 E$ ). Together, these results reveal that novel object exploration facilitates the induction of hippocampal CA1 LTD, without affecting hippocampal CA1 LTP in freely moving animals.

It is well documented that the induction of hippocampal CA1 LTD is dependent on activation of the NMDA-subtype glutamate receptor (NMDAR) (Dudek and Bear, 1992; Mulkey and Malenka, 1992; Malenka and Nicoll, 1993). Moreover, recent studies have also supported a requirement of the GluN2Bcontaining subpopulation of NMDARs in vitro and in vivo (Liu et al., 2004; Fox et al., 2006; Wang et al., 2006; Ge et al., 2010). However, whether GluN2B-containing NMDAR activation is required for the induction of the novelty exploration-facilitated hippocampal CA1 LTD has previously not been examined. Therefore, we next examined the potential involvement of GluN2B receptor by applying Ro25-6981, a specific GluN2B antagonist, before novel object exploration and LFS. As shown in Figure $2 A$, LFS in combination with novelty exploration, while producing robust LTD in saline-treated control rats (saline: $n=$ $4,69.9 \pm 4.1 \%, p=0.002$ vs baseline; Fig. $2 A$ ), failed to induce LTD in the Ro25-6981-treated rats (Ro25-6981: $n=7,97.6 \pm$ $2.9 \%, p=0.309$ vs baseline, $p<0.001$ vs saline; Fig. $2 A)$. We then determined whether a facilitated endocytosis of AMPA-subtype glutamate receptors (AMPARs), a process required for the expression of various forms of LTD in several brain regions (Collingridge et al., 2010), may also be a critical mechanism in the expression of this novelty-facilitated hippocampal LTD. To this end, we applied Tat-GluA2 3 y peptide, a membrane-permeable peptide inhibitor that prevents the expression of various forms of LTD by interfering with the facilitated endocytosis of AMPARs without affecting either basal synaptic transmission or LTP (Ahmadian et al., 2004; Brebner et al., 2005; Van den Oever et al., 2008; Ge et al., 2010). Systemic administration of Tat-GluA2 3 Y (Tat-GluA2 $2_{3 \mathrm{Y}}: n=6,97.5 \pm 3.2 \%, p=0.278$ vs baseline, $p<$ 0.001 vs sTat-GluA2 3 ; Fig. $2 A$ ), but not its scrambled control peptide ( $s$ Tat-GluA2 $2_{3 \mathrm{Y}}: n=5,64.9 \pm 3.7 \%, p=0.001$ vs baseline; Fig. $2 A), 1 \mathrm{~h}$ before LFS and novel object exploration prevented hippocampal CA1 LTD expression.

These results suggest that the hippocampal CA1 LTD facilitated by novel object exploration requires activation of GluN2Bcontaining NMDA receptors in its induction and GluA2-dependent AMPAR endocytosis in its expression and maintenance. If that is the case, one would expect that application of Tat-GluA2 $2_{3 Y}$ peptide, but not Ro25-6981, immediately after the LTD induction would remain effective in preventing LTD expression. Consistent with our prediction, application of Ro25-6981 or vehicle (saline) immediately after LFS failed to affect novel object exploration-facilitated LTD (saline: $n=3,69.7 \pm 0.7 \%, p<0.001$ vs baseline; Ro: $n=4,73.1 \pm 3.1 \%$, $p=0.002$ vs baseline, $p=0.858$ vs saline; Fig. $2 B$ ). However, TatGluA2 $2_{3 Y}$ (but not its scrambled control sTat-GluA2 $2_{3 Y}$ peptide) applied immediately after LFS, while having little effect on the immediate postdepression, prevented the expression/maintenance of LTD (sTat-GluA2 $2_{3 Y}: n=3,71.9 \pm 3.2 \%, p=0.007$ vs baseline; Tat-GluA2 ${ }_{3 \mathrm{Y}}: n=5,99.7 \pm 2.3 \%, p=0.403$ vs baseline, $p<0.001$ vs sTat-GluA2 ${ }_{3 Y}$; Fig. $2 B$ ). 
A
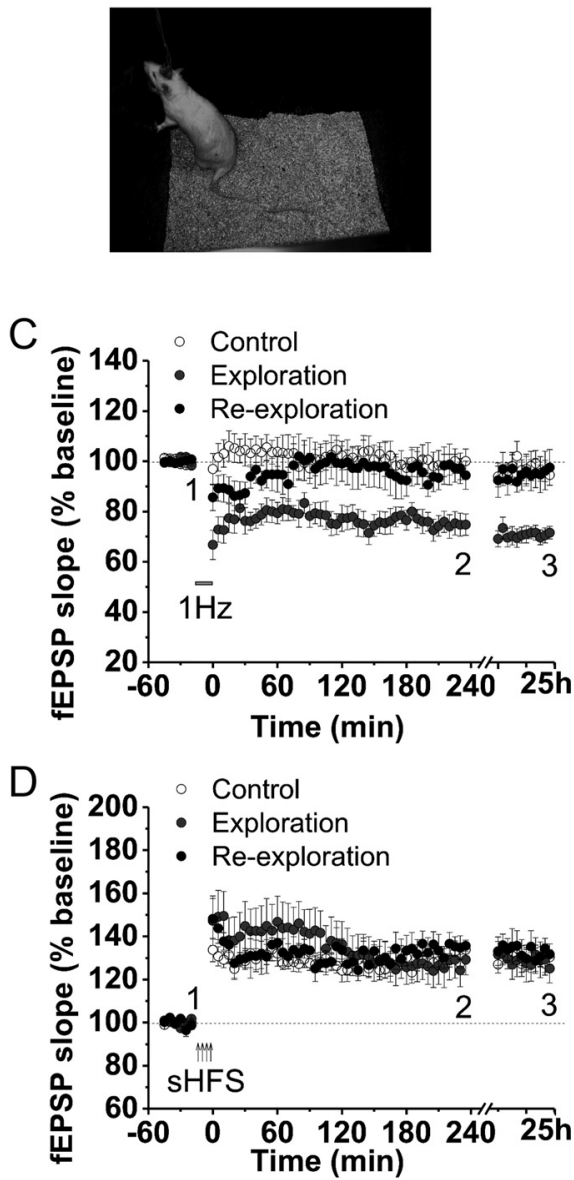

E

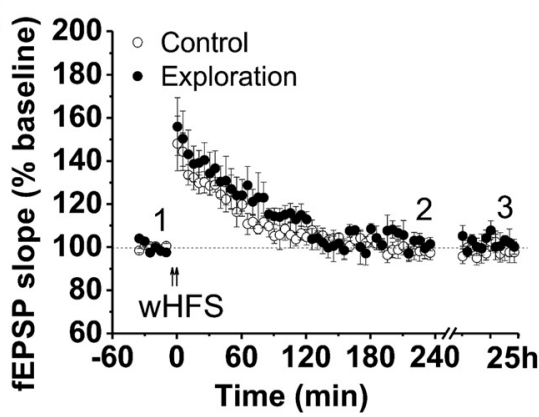

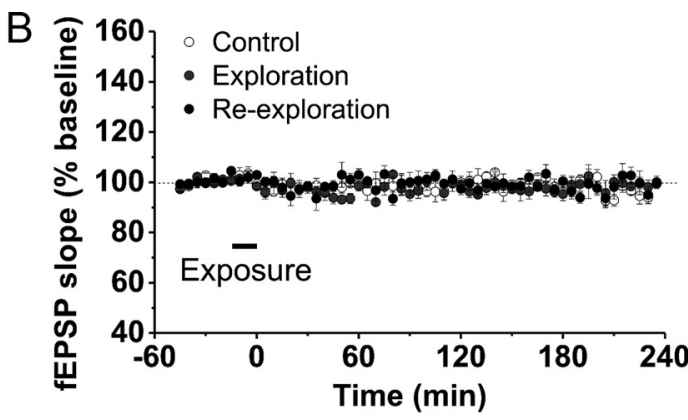
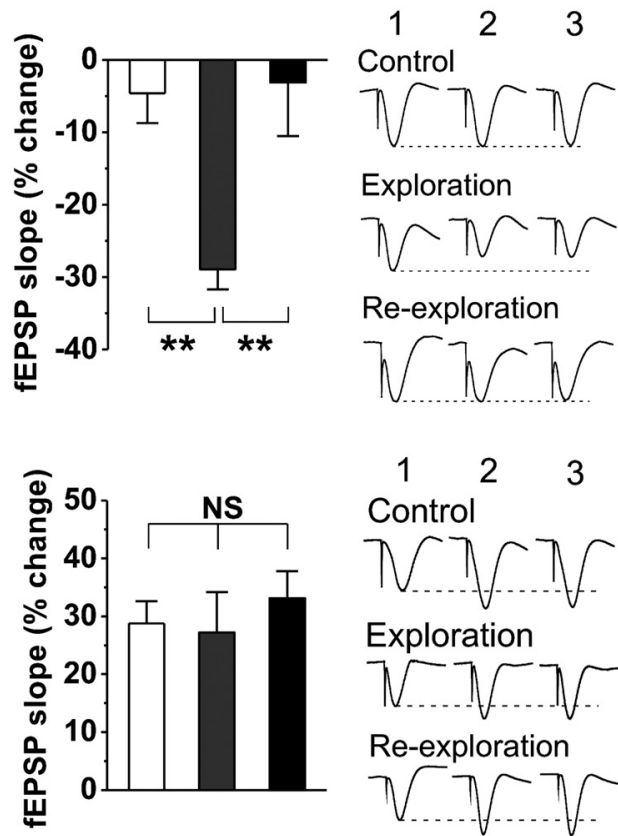

123

Control

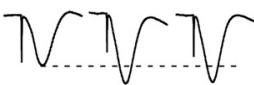

Exploration

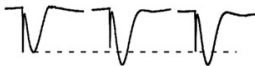

Re-exploration
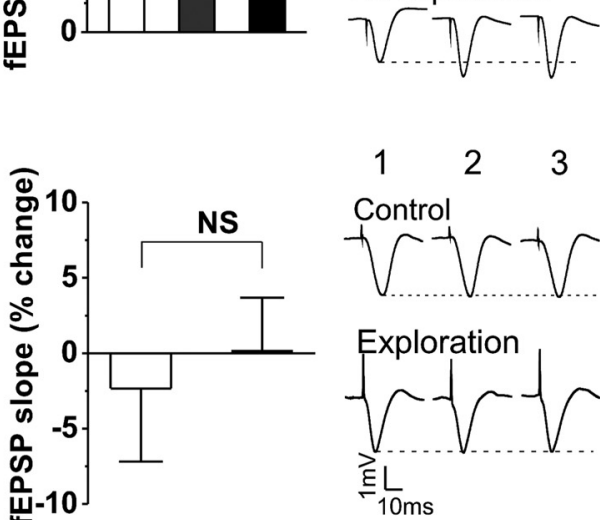

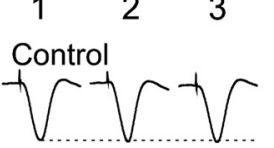

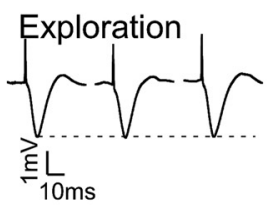

Figure 1. Novel object exploration facilitates hippocampal CA1 LTD in freely moving rats. $A$, Representative photograph of a freely moving rat during a recording session. $B$, Novel object exploration does not affect the basal level of synaptic transmission. The plot of normalized slopes offEPSPs show thata 15 min period exploration of eithernovel (exploration; $n=4$ ) or familiar (reexploration; $n=4$ ) objects (indicated by the black bar) has does not affect basal level of fEPSPs, compared with that recorded from naive animals (control; $n=4)$. No difference among groups, post hoc Fisher's test after ANOVA $\left(F_{(2,9)}=0.015 ; p=\right.$ 0.985). C, Novel (but not familiar) object exploration enables LFS to induce hippocampal CA1 LTD of fEPSPs. The plots (left) of normalized slopes of fEPSPs show that the hippocampal LTD could only be induced by LFS (900 pulses at $1 \mathrm{~Hz}$ ) in animals simultaneously allowed to explore novel objects (exploration; $n=7$ ), but not in naive animals $($ control; $n=8)$ or in animals which simultaneously reexplore objects that the animals had exposed to for a $15 \mathrm{~min}$ period $24 \mathrm{~h}$ before the LFS (reexploration; $n=5$ ). The bar graph (center) summarizes the average percentage change of fEPSP slope immediately before and $24 \mathrm{~h}$ after $\mathrm{LFS}$, and corresponding representative traces are shown on the right. ${ }^{* *} p<0.01$, post hoc Fisher's test after ANOVA $\left(F_{(2,17)}=8.838 ; p=0.002\right)$. D , Novelty exploration does not affect a sHFS protocol $(4 \times 30$ pulses at $100 \mathrm{~Hz}$, with an intertrain interval of $5 \mathrm{~min}$ ) induced hippocampal LTP in freely moving rats. The effects of novel (exploration; $n=7$ ) and familiar (reexploration; $n=5)$ exploration, along with naive controls (control; $n=7$ ) on LTP are summarized in the plots (left) and bar graph (center). Representative traces taken at the time points indicated in the plots are shown on the right. One-way ANOVA shows no statistically significant difference (NS) among these groups $\left(F_{(2,16)}=0.252 ; p=0.780\right)$. E, Novelty exploration has no effect on hippocampal early LTP. The eLTP, which only lasted for $<1.5 \mathrm{~h}$, was induced by a wHFS protocol $(2 \times 30$ pulses at $100 \mathrm{~Hz}$, with an intertrain interval of $5 \mathrm{~min}$ ). The effects of novel object exploration during wHFS on eLTP (exploration; $n=4)$, compared with eLTP induced with wHFS from naive animals (control; $n=4$ ) are summarized in the plots (left) and bar graph (center). Representative traces are shown on the right. NS, No statistically significant difference, post hoc Fisher's test after ANOVA $\left(F_{(1,6)}=0.136 ; p=0.725\right)$. Error bars indicate SEM.

Previous studies have shown that metabotropic glutamate receptors (mGluRs) play a critical role in the induction of LTD in vivo (Manahan-Vaughan, 1998) and in vitro (Mellentin and Abraham, 2001) via a priming effect. To determine whether
mGluR signaling has a critical role in novelty enhancement in LTD production, we next used a nonselective group I/group II mGluR antagonist MCPG (500 nmol in $5 \mu \mathrm{l}$; intracerebroventricular infusion) $1 \mathrm{~h}$ before LFS. Here, we found that the appli- 

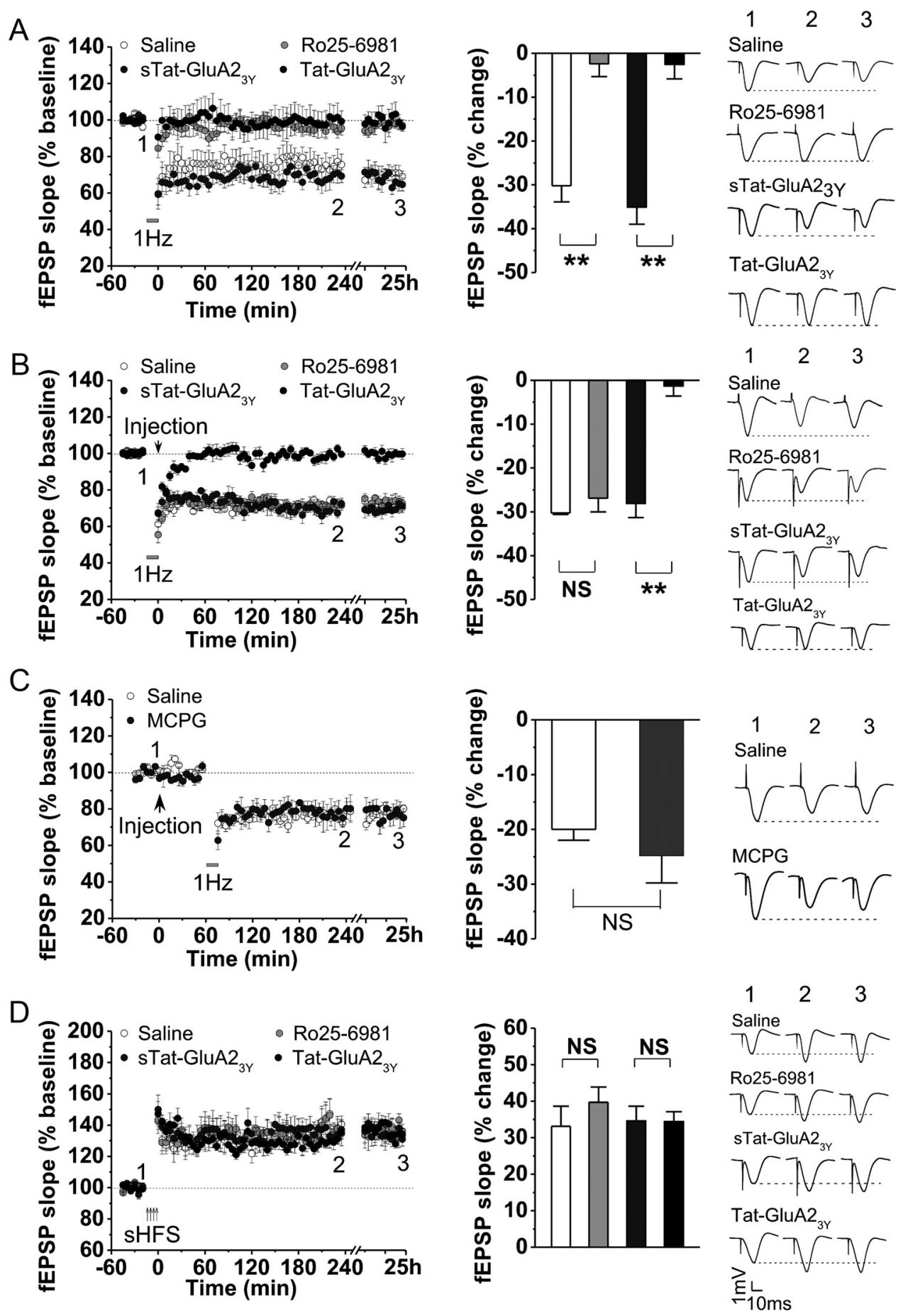

Figure 2. Activation of GluN2B-containing NMDARs and GluA2-dependent AMPAR endocytosis are required for the induction and expression of the novel object exploration-facilitated hippocampal CA1 LTD in freely moving rats, respectively. $A$, The plot (left) and bar graph (center) of fEPSPs show that systemic application (intraperitoneally) of GluN2B-specific inhibitor Ro25-6981 (6 mg/kg; $n=7$ ) or AMPAR endocytosis inhibitor Tat-GluA2 ${ }_{3 Y}(3 \mu \mathrm{mol} / \mathrm{kg} ; n=6)$, but not saline $(n=4)$ or sTat-GluA2 $3 \mathrm{Y}(3 \mu \mathrm{mol} / \mathrm{kg} ; n=5)$, could prevent the hippocampal LTD. Representative traces are shown on the right $\left(F_{(3,18)}=\right.$ 26.330; $p<0.001 ;{ }^{* *} p<0.01$ ). B. The plot (left) and bar graph (center) of fEPSPs show that only systemic application (intraperitoneally) of Tat-GluA2 3 peptide ( $\left.3 \mu \mathrm{mol} / \mathrm{kg} ; n=3\right)$, but not Ro25-6981 (6 $\mathrm{mg} / \mathrm{kg} ; n=4)$ or saline $(n=3)$ or scrambled Tat-GluA2 3 Y peptide $(3 \mu \mathrm{mol} / \mathrm{kg} ; n=5)$ treatment given immediately after LFS could prevent the hippocampal LTD $\left(F_{(3,11)}=27.473 ; p<0.001 ; * * p<0.01\right)$. C, The plot (left) and bar graph (center) of fEPSPs show that the application of group I/group II mGluRantagonistMCPG ( $500 \mathrm{nmol}$ in $5 \mu$ l; intracerebroventricular infusion; $n=4)$ or saline $(n=4) 1 \mathrm{~h}$ before LFS delivery have no effect on basal fEPSPs and the LTD facilitated by novel object exploration $\left(F_{(1,6)}=0.645 ; p=0.452\right)$. $\boldsymbol{D}$, The plot (left) and bar graph (center) of fEPSPs show that systemic application (intraperitoneally) of neither Ro25-6981 ( $n=5$ ) nor Tat-GluA2 3 peptide $(n=5)$ nor their controls (saline, $n=5$; or sTat-GluA2 $3 y, n=5$ ) affected sHFS-induced hippocampal CA1 LTP in freely moving rats $\left(F_{(3,16)}=0.558 ; p=0.650\right)$. Representative traces are shown on the right. NS, No statistically significant difference. Error bars indicate SEM.

cation of MCPG did not significantly alter either the basal synaptic transmission or the novel object exploration-facilitated LTD (MCPG: $n=4,75.2 \pm 5.0 \%, p=0.013$ vs baseline, $p=$ 0.114 vs saline; Fig. $2 C$ ), compared with vehicle (saline: $n=4$,
$80.1 \pm 2.0 \%, p=0.001$ vs baseline; Fig. $2 C$ ). These results suggest that the exploration of novel objects actually produces NMDARdependent LTD but does not produce an mGluR-dependent novelty priming phenomenon. 
A
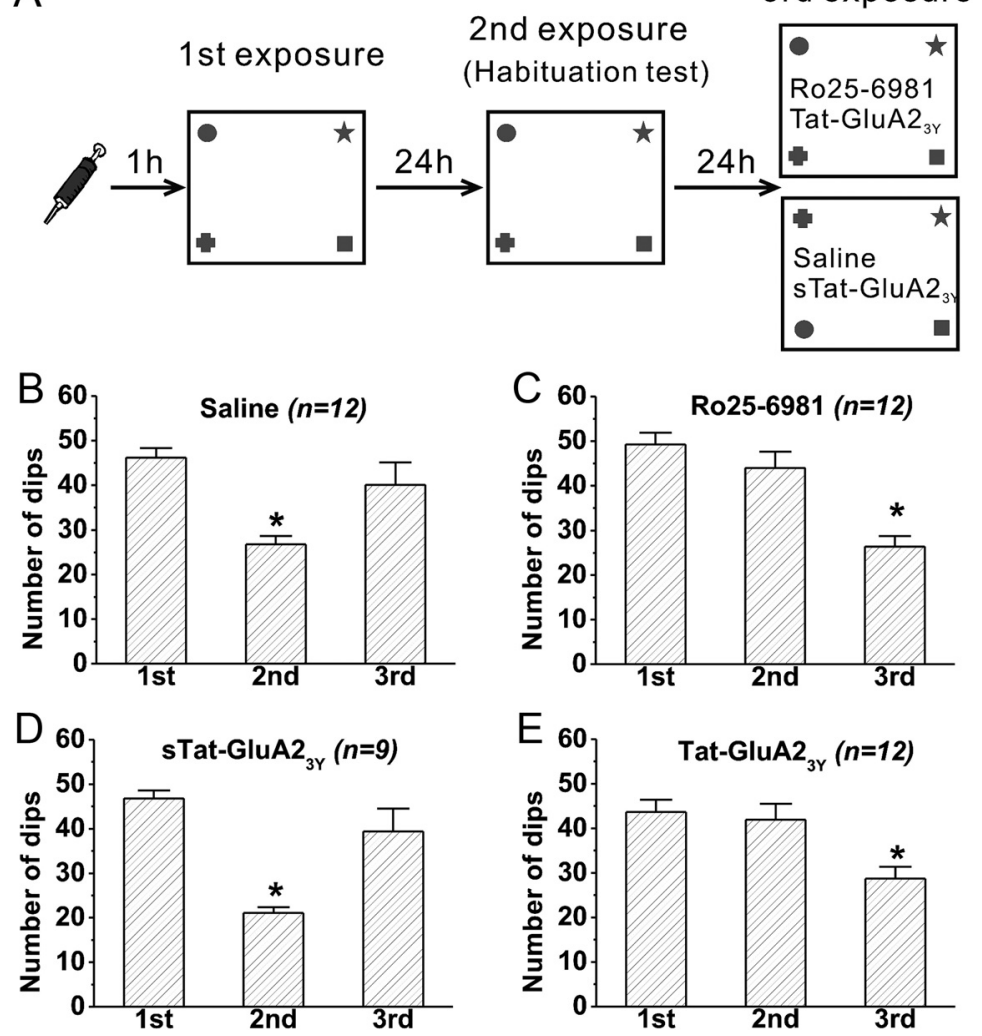

3rd exposure

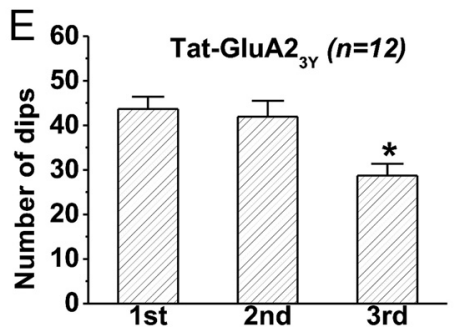

Figure 3. Inhibition of hippocampal CA1 LTD impairs novelty acquisition. $A$, Diagrams illustrating the experimental protocol for three consecutive 15 min explorations separated by a $24 \mathrm{~h}$ test interval. One of LTD inhibitors or controls was systemic applied via intraperitoneal injection $1 \mathrm{~h}$ before the first exposure to novel objects. $\boldsymbol{B}-\boldsymbol{E}$, Inhibition either LTD induction with R025-6981 or LTD expression with Tat-GluA2 $2_{3 \gamma}$ impairs novelty acquisition. One-way ANOVA followed post hoc Fisher's test analysis, first versus second or third; ${ }^{*} p<0.05$ and ${ }^{* *} p<0.01$. Rats receiving saline $(B)\left(F_{(2,33)}=4.995 ; p=0.013\right)$ or sTat-GluA2 ${ }_{3 \gamma}$ control peptide (D) $\left(F_{(2,24)}=11.082 ; p<0.001\right)$ did not affect the novelty acquisition during the first exposure as the animals recognized the same object configuration during the second exposure showing decreased numbers of head dips (habituation; first vs second exposure: $p<0.05$ for saline and $p<0.001$ for sTat-GluA2 $2_{3 Y}$ ) and recognized the object rearrangement as novelty configuration during the third exposure resulting in a new novelty acquisition similar to that with first exposure. In contrast, animal receiving either Ro25-6981 (C) $\left(6 \mathrm{mg} / \mathrm{kg} ; F_{(2,33)}=11.009 ; p<0.001\right)$ or Tat-GluA2 ${ }_{3 Y}(\boldsymbol{E})(3 \mu \mathrm{mol} / \mathrm{kg}$; $\left.F_{(2,33)}=26.924 ; p<0.001\right)$ before the first exposure showed no novelty acquisition during the first exposure as the animals failed to recognize the same object configuration, yielding a similar numbers of head dip during second exposure, but they did acquire the novel information having significantly fewer numbers of head dips during the third exposure to the same object configuration. Error bars indicate SEM.

To further ascertain that these two LTD inhibitors have no effect on either induction or expression of LTP, we next systemically applied these two drugs, along with controls, $1 \mathrm{~h}$ before sHFS delivery. As shown in Figure 2D, neither Ro25-6981 (saline: $n=5,133.1 \pm 5.3 \%, p=0.002$ vs baseline; Ro: $n=5,139.7 \pm$ $3.1 \%, p<0.001$ vs baseline, $p=0.474$ vs saline; Fig. $2 D$ ) nor Tat-GluA2 $2_{3 \mathrm{Y}}$ peptide (sTat-GluA2 $3 \mathrm{Y}: n=5,134.6 \pm 3.7 \%, p<$ 0.001 vs baseline; Tat-GluA2 3 Y $: n=5,134.4 \pm 2.9 \%, p<0.001$ vs baseline, $p=0.279$ vs sTat-GluA2 ${ }_{3 Y}$; Fig. $2 D$ ) affected hippocampal CA1 LTP in freely moving rats under our experimental conditions. Together, these results demonstrate that activation of GluN2B-containing NMDARs and consequent AMPAR endocytosis are required for the induction and expression of novel object exploration-facilitated hippocampal CA1 LTD, respectively. Moreover, our results also suggest that Ro25-6981 and Tat-GluA2 3 Y peptide are two structurally and mechanistically distinct LTD specific inhibitors that may be used to probe a causal role of novelty exploration-facilitated LTD in behavioral animals.

\section{Novelty exploration-facilitated hippocampal LTD is required for novelty acquisition}

It has been accepted that animal exploratory behavior represents a form of information storage and that object recognition learning has taken place during exposure to the novel objects (File and Wardill, 1975; Platel and Porsolt, 1982). However, whether the new object exploration-facilitated hippocampal LTD has a causal role in the hippocampaldependent novelty acquisition learning remains largely undefined due to a lack of specific inhibitors to LTD. Therefore, we next examined the role of hippocampal LTD in novelty acquisition using these two specific LTD inhibitors, Ro25-6981 and Tat-GluA2 ${ }_{3 \mathrm{Y}}$ peptide (Fig. 3).

Hippocampal-dependent novelty acquisition in the rat was examined using experimental procedures illustrated in Figure $3 A$. The number of head dips toward each object was counted during a 15 min exposure period for three times with an interval of $24 \mathrm{~h}$, and vehicle or inhibitors were systemically administrated via an intraperitoneal injection $1 \mathrm{~h}$ before the first exploration trial (Fig. 3A). The saline$(n=12, p=0.017$ for first vs second trial; Fig. $3 B)$ and sTat-GluA2 $2_{3 Y}$ peptide- $(n=$ $9, p<0.001$ for first vs second trial; Fig. $3 D$ ) treated groups showed significantly less head dips when reexposed to the objects during second exposure compared with their performance in the first trial. Thus, learning had occurred during the first exposure as the animals were able to remember their environment during second trial performed $24 \mathrm{~h}$ after exploration. However, when objects were rearranged for the third trial, the number of head dipping in both groups significantly increased in comparison with that of second trial, reaching a level similar to that of the first trial (saline: $n=12, p=$ 0.925 for first vs third trial, $p=0.042$ for second vs third trial; sTat-GluA2 ${ }_{3 \mathrm{Y}}: n=9, p=0.307$ for first vs third trial, $p=0.013$ for second vs third trial; Fig. $3 B, D$ ). These results suggest that the reduction of head dipping observed on the second trial was a result of habituation due to the memory of the object-place configuration of the first trial and that the rearrangement of the familiar objects on the third trial put the animals into a novel environment, and thereby brought the exploration behavior back to the level that was seen on the first trial. On the contrary, animals pretreated with Ro25-6981 (Ro25-6981: $n=12, p=0.925$ for first vs second trial; Fig. $3 C$ ) or Tat-GluA2 ${ }_{3 Y}$ peptide (TatGluA2 $_{3 \mathrm{Y}}: n=12, p=0.794$ for first vs second trial; Fig. $3 E$ ) showed similar head-dipping levels when reexposed to the same object configuration environment on the second trial compared with their performance in the first trial, suggesting that Ro256981 or Tat-GluA2 ${ }_{3 Y}$ injection before the first trial prevented the memory formation of new object-place configuration during the first trial and consequently, a new object configuration learning 
remained to take place during the second exposure. Consistent with the learning occurrence during the second, but not first trial following these animals, numbers of head dipping were dramatically decreased when Ro25-6981- or Tat-GluA2 $3 \mathrm{Y}^{-}$ treated groups were reexposed to the same object configuration on the third trial (Ro25-6981: $n=12, p=0.002$ for first vs third trial, $p<0.001$ for second vs third trial; Tat-GluA2 $2_{3 \mathrm{Y}}: n=12, p<0.001$ for first vs third trial, $p<0.001$ for second vs third trial; Fig. $3 C, E)$. Thus, these results demonstrate that novelty exploration-facilitated hippocampal LTD causally contributes to hippocampus-dependent novelty acquisition.

\section{Novelty exploration enhances memory by producing hippocampal LTD}

In one of our most recent studies, we showed that induction of LTD may have an essential role in spatial memory consolidation (Ge et al., 2010). Since our aforementioned results strongly suggested that novel object exploration facilitated the induction of hippocampal LTD, we hypothesized that, by helping the consolidation of newly formed memory, the novel object exploration facilitated hippocampal LTD may play a critical role in novel environment-induced memory enhancement. We next tested this hypothesis using a well characterized hippocampus-dependent learning task, IA training (Fig. 4A). To determine the role of novel object explorationfacilitated LTD in novel environment-induced memory enhancement, we used two different intensities of footshock: a weak footshock IA (wIA) $(0.2 \mathrm{~mA}, 2 \mathrm{~s}$ ) and a strong footshock IA (sIA) $(0.4 \mathrm{~mA}, 2 \mathrm{~s})$, and memory tests measuring the latency of the animals from the onset of shock to step into the dark compartment were performed at 1 and $24 \mathrm{~h}$ after the training. As shown in Figure $4 B$, wIA training could only produce a STM $(n=9,54.9 \pm 16.0$ s for test, $p=0.012$ vs training; Fig. $4 B$ ) that was only retrieved within $1 \mathrm{~h}$, but $\operatorname{not} \operatorname{LTM}(n=12,10.8 \pm 2.8$ s for test, $p=0.136$ vs training; Fig. $4 B)$ that was only retrieved over $24 \mathrm{~h}$, after the training. In contrary, sIA training could produce a LTM that lasted $>24 \mathrm{~h}$ (Fig. $4 D$ ). Consistent with our prediction, we found that wIA footshock, which failed to produce any observable effect on the latency to step into the dark compartment at $24 \mathrm{~h}$ in naive animals (control: $n=12,10.8 \pm 2.8 \mathrm{~s}$ for test, $p=0.136$ vs training; Fig. $4 C$ ), reliably and significantly prolonged the latency in animals that were exposed to a novel object exploration for a period of $15 \mathrm{~min}$ just $1 \mathrm{~h}$ before IA training (exploration: $n=9,60.7 \pm 12.6 \mathrm{~s}$ for test, $p=0.007$ vs training, $p<0.001$ vs control; Fig. 4C). This memory enhancement is novel object exploration related, as the prolonged latency was not observed in rats receiving a $15 \mathrm{~min}$ of familiar object exploration $1 \mathrm{~h}$ before the weak IA training (reexploration: $n=9,17.7 \pm 7.3 \mathrm{~s}$ for test, $p=0.295 \mathrm{vs}$ training, $p=0.824$ vs control, $p=0.005$ vs exploration; Fig. $4 C$ ). Thus, prior novel object exploration was able to enhance the memory formation produced by wIA training, thereby converting STM into LTM. In contrary, the novel object exploration had no effect on LTM formation induced by the sIA training, as both naive group (control: $n=8,218.8 \pm 48.3$ s for test, $p=0.002$ vs training; Fig. $4 D$ ) and group that received $15 \mathrm{~min}$ novel object exploration $1 \mathrm{~h}$ before
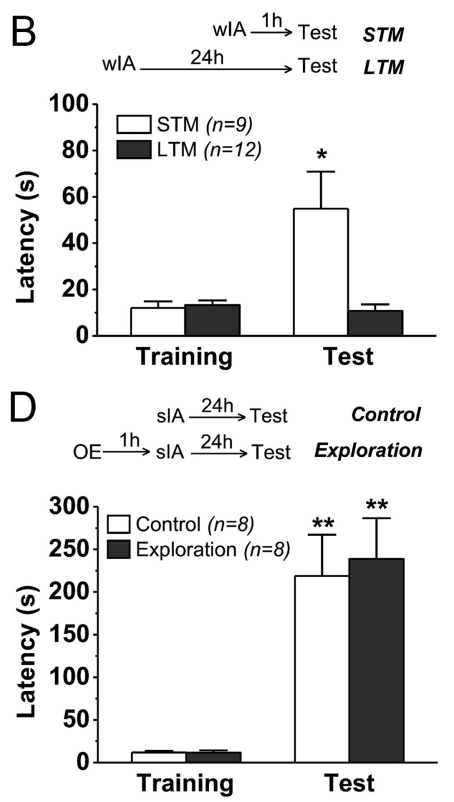

Figure 4. Novel object exploration enhances memory by promoting the conversion of STM into LTM. A, Basic apparatus used in the single-trial acquisition of IA test. During the training session, when the animal steps into the dark compartment, it will receive either a weak (wIA) $(0.2 \mathrm{~mA}$ for $2 \mathrm{~s})$ or strong $(\mathrm{s} \mid \mathrm{A})(0.4 \mathrm{~mA}$ for $2 \mathrm{~s})$ footshock, and the latency for the animal to step into the dark STM thas for for tring STM into LTM. Animals receiving a 15 min period of novel object exploration (OE) $1 \mathrm{~h}$ before wIA (exploration) showed a significant in latency compared with control animals (control) measured $24 \mathrm{~h}$ later. This novel object exploration memory ancement was lost in animals that had been exposed to the same objects $24 \mathrm{~h}$ before wIA training (reexploration) $\left(F_{(2,27)}=\right.$

IA training (exploration: $n=8,238.8 \pm 47.8$ s for test, $p=0.001$ vs training, $p=0.394$ vs control; Fig. $4 D$ ) showed a similar latency to step into dark compartment during test sessions performed $24 \mathrm{~h}$ after the training. These results suggest that the novel object exploration can enhance memory by converting STM into LTM.

LTD production has recently been shown to increase the consolidation of the memory learned after LTD production (Ge et al., 2010). Since novel object exploration facilitates the induction of hippocampal LTD, it is reasonable to speculate that this LTD may have a critical role in mediating the novel object exploration-dependent STM and LTM conversion. This was next examined using the aforementioned two structurally and mechanistically distinct LTD inhibitors. We first bilaterally injected Ro25-6981 or Tat-GluA2 3 Y peptide into the dorsal hippocampus $30 \mathrm{~min}$ before novel object exploration via a cannula chronically implanted $7-10 \mathrm{~d}$ before the experiment. As shown in Figure 5, following the wIA training, a significant increase in the latency to step into the dark compartment at $24 \mathrm{~h}$ was only observed in animals receiving either saline $(n=8,67.1 \pm 17.4 \mathrm{~s}$ for test, $p=0.009$ vs training; Fig. $5 A)$ or sTat-GluA2 ${ }_{3 \mathrm{Y}}(n=8$, $71.3 \pm 19.0 \mathrm{~s}$ for test, $p=0.008$ vs training; Fig. $5 B$ ), but not in animals receiving either Ro25-6981 $(n=8,16.4 \pm 3.1$ s for test, $p=$ 0.258 vs training, $p=0.017$ vs saline; Fig. $5 A$ ) or Tat-GluA2 3 Y peptide ( $n=8,13.4 \pm 6.6 \mathrm{~s}$ for test, $p=0.326$ vs training, $p=0.017$ vs sTat-GluA2 $2_{3 \mathrm{Y}}$; Fig. $5 B$ ), suggesting an essential role of LTD production in the novelty exploration-induced STM-LTM conversion. Since Ro25-6981 and Tat-GluA2 ${ }_{3 Y}$ peptide inhibited induction and expression of LTD (Fig. $2 A, B$ ), respectively, we predicted that, if the production of LTD was critically required for the STM-to-LTM conversion, infusion of Ro25-6981 immediately after the novel object exploration should not affect the STM-LTM conversion, as it failed to prevent the expression of LTD when given after the LTD induc- 

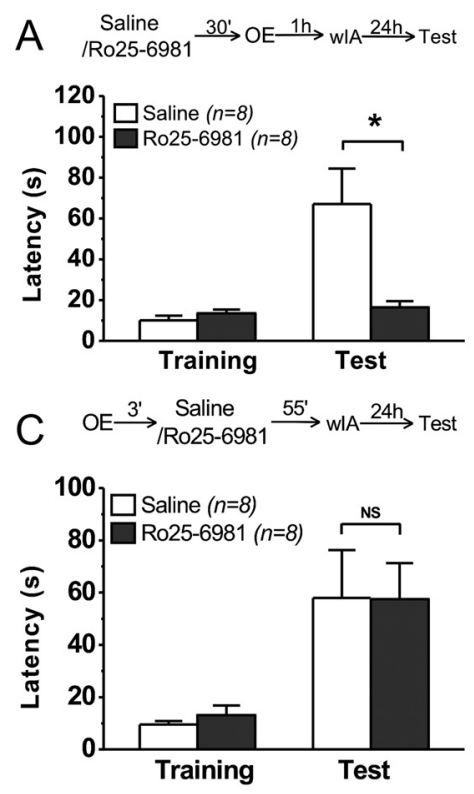

E

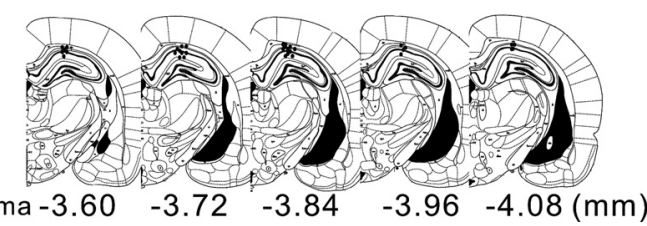

Figure 5. Hippocampal LTD is required for the novelty exploration-induced memory enhancement. $\boldsymbol{A}, \boldsymbol{B}$, Respective inhibition of LTD induction and expression by bilateral infusion of R025-6981 $(\boldsymbol{A})\left(0.5 \mathrm{nmol} / \mu \mathrm{l}\right.$ per side; $\left.F_{(1,14)}=7.257 ; p=0.017\right)$ and Tat-GluA2 ${ }_{3 Y}(\boldsymbol{B})\left(100 \mathrm{pmol} / \mu \mathrm{l}\right.$ per side; $\left.F_{(1,14)}=7.273 ; p=0.017\right)$ into the hippocampus $30 \mathrm{~min}$ before novel object exploration $(\mathrm{OE})$ prevented the novel object exploration-promoted conversion of wIA-induced STM into LTM, whereas infusion of their respective controls saline $(\boldsymbol{A})$ or sTat-GluA2 ${ }_{3 Y}(\boldsymbol{B})$ had no effect. $\boldsymbol{C}, \boldsymbol{D}$, Bilateral intrahippocampal infusion of Ro25-6981 or saline $(\boldsymbol{C})\left(F_{(1,14)}\right.$ $=0.004 ; p=0.984 ; \mathrm{NS}$, no significant difference) immediately after the novel object exploration fails to prevent the novelty exploration-induced memory enhancement (i.e., wIA was still capable of producing LTM), whereas a similar intrahippocampal infusion of Tat-GluA2 $2_{3 Y}$ (but not its control sTat-GluA2 ${ }_{3 Y}$ ) remains effective in preventing the memory enhancement $(\boldsymbol{D})\left(F_{(1,14)}=\right.$ $5.290 ; p=0.037) . E$, Diagrams illustrating the placements of the infusion sites. The plates were adopted from Paxinos and Watson (2004), and their distances posterior from bregma are indicated in millimeters. ${ }^{*} p<0.05$. Error bars indicate SEM.

tion (Fig. 2B; Ro25-6981). However, infusion of Tat-GluA2 $2_{3 Y}$ immediately after the novel object exploration should prevent the conversion of STM to LTM, since when given immediately after induction, it remained effective in preventing the expression of LTD (Fig. $2 B$; Tat-GluA2 3 Y). Consistent with our predictions, we indeed found that bilateral intrahippocampal infusion of TatGluA2 $2_{3 Y}$ peptide, but not Ro25-6981 or other controls, immediately ( $\sim 3$ min for dummy cannulas removal and injection cannulas insertion) after the novel object exploration prevented the conversion of STM to LTM (saline: $n=8,58.0 \pm 18.3$ s for test, $p=0.018$ vs training; Ro: $n=8,57.5 \pm 13.8 \mathrm{~s}$ for test, $p=0.010$ vs training, $p=$ 0.984 vs saline; sTat-GluA2 ${ }_{3 Y}: n=8,63.5 \pm 18.3$ s for test, $p=0.018$ vs training; Tat-GluA2 $2_{3 \mathrm{Y}}: n=8,16.8 \pm 5.1 \mathrm{~s}$ for test, $p=0.092 \mathrm{vs}$ training, $p=0.037$ vs sTat-GluA2 ${ }_{3 Y}$; Fig. $5 C, D$ ). Together, these results reveal that the expression of hippocampal LTD is critically required for novel object exploration mediated memory enhancement (i.e., the conversion of STM into LTM).

\section{Expression of hippocampal LTD is sufficient to promote the conversion of STM into LTM}

Recent studies have revealed that the hippocampal CA1 LTD in adult rats, although being difficult to produce with standard LFS protocol ( $1 \mathrm{~Hz}$ for $15 \mathrm{~min}$ ), could be reliably induced with stron- ger induction protocols such as the paired burst protocol (200 pairs of two-pulse bursts at one pair, with an interval of 2.5 ms between pulses and $10 \mathrm{~ms}$ between bursts) (Thiels et al., 1996; Staubli and Scafidi, 1997; Ge et al., 2010). Moreover, this paired burst induced LTD in freely moving rats, similar to the novel object exploration enabled LTD, is also mediated by a mechanism requiring GluN2B activation and GluA2-dependent AMPAR endocytosis in its induction and expression, respectively (Ge et al., 2010). Therefore, to determine whether the expression of LTD was sufficient to account for the novel object exploration-mediated memory enhancement, we then examined whether the induction of hippocampal LTD by paired burst could mimic the novel object exploration, sufficiently promoting the conversion of STM into LTM. As shown in Figure $6 A$, consistent with previous reports (Thiels et al., 1996; Staubli and Scafidi, 1997; Ge et al., 2010), pairedburst stimulation reliably induced hippocampal CA1 LTD in freely moving rats (test pulse: $n=8,100.5 \pm 0.9 \%, p=0.477$ vs baseline; paired burst: $n=8,73.9 \pm$ $3.5 \%, p<0.001$ vs baseline, $p<0.001$ vs test pulse; Fig. $6 \mathrm{~A}$ ). Two hours after the LTD induction, the rats received a wIA training. As we predicted, production of LTD on either side of the hippocampi before the wIA training significantly increased the latency to step into the dark compartment measured $24 \mathrm{~h}$ after the training (test pulse: $n=8,13.3 \pm 1.7 \mathrm{~s}$ for test, $p=0.106$ vs training; paired burst: $n=8,61.8 \pm 22.8 \mathrm{~s}$ for test, $p=0.036 \mathrm{vs}$ training, $p=0.034$ vs test pulse; Fig. $6 B$ ), being sufficient to convert the wIA-induced STM into LTM. To further support the sufficiency of LTD in mimicking novel object exploration in memory enhancement, we also examined effects of glutamate transporter inhibitor as recent evidence indicating that hippocampal LTD in adult rats could also be reliably induced with the standard LFS protocol after blocking glutamate transport activity with the transporter inhibitor DL-TBOA in vitro (Yang et al., 2005) and in vivo (Wong et al., 2007). Consistent with these previous reports, we observed that intracerebroventricular infusion of DL-TBOA $(n=5,73.3 \pm 1.8 \%, p<0.001$ vs baseline, $p<0.001$ vs saline; Fig. $6 C$ ), but not the same volume of saline $(n=4,98.9 \pm 2.4 \%, p=0.221$ vs baseline; Fig. $6 C)$ enabled the LFS to produce LTD in freely moving rats, without affecting basal synaptic transmission or eLTP induced by wHFS (saline: $n=4,101.7 \pm 1.8 \%, p=0.098$ vs baseline; DL-TBOA: $n=$ $4,100.2 \pm 1.5 \%, p=0.409$ vs baseline, $p=0.368$ vs saline; Fig. $6 D)$. In supporting the sufficiency of LTD to mediate the memory enhancement, we found that the intrahippocampal infusion of DL-TBOA, but not saline, converted the wIA-induced STM into LTM (saline: $n=8,17.8 \pm 3.6$ s for test, $p=0.104$ vs training; DL-TBOA: $n=9,51.0 \pm 14.4$ s for test, $p=0.010$ vs training, $p=0.040$ vs saline; Fig. $6 E$ ). 


\section{Discussion}

In the present study, we confirm that novelty exploration facilitates the induction of hippocampal CA1 LTD in freely moving rats and demonstrate that the induction and expression of this novelty-promoted LTD are dependent on the activation of GluN2B-containing NMDARs and GluA2-dependent AMPARs endocytosis, respectively. By correspondingly blocking the induction and expression of this LTD with two inhibitors that differ by structure and mechanism of action, we are able to show that hippocampal LTD is not only required for novelty acquisition but is also critical for novelty exploration enhancement of memory. More importantly, through promotion of hippocampal LTD induction via selective inhibition of glutamate transporter activity, we also demonstrate that facilitation of hippocampal LTD is sufficient to mediate the memory enhancement by novelty exploration. We have therefore provided compelling evidence for the role of novelty exploration-promoted hippocampal LTD in mediating the novelty acquisition, and more importantly, the memory enhancement by novelty exploration.

It has been well documented that hippocampal LTD is difficult to be induced by the classical LFS protocol ( 900 pulses at $1 \mathrm{~Hz}$ ) in adult rats (Dudek and Bear, 1993; Wagner and Alger, 1995; Staubli and Scafidi, 1997; Kemp and ManahanVaughan, 2004; Xiong et al., 2004). However, as demonstrated previously (Manahan-Vaughan and Braunewell, 1999) and convincingly replicated here, LFS can reliably induce hippocampal LTD in freely moving rats when the animals are allowed to explore novel, but not familiar (reexploring), objects during LFS. However, we found that neither novel object exploration nor familiar object exploration (reexploration) during HFS affected the ability of HFS to induce hippocampal LTP. Thus, under our experimental conditions, the novel object exploration specifically promotes the induction of hippocampal LTD and does not affect basal synaptic transmission or the production of LTP. It is interesting to note that previous report showed that LTP was depotentiated by exploration of a novel environment in freely moving rats (Xu et al., 1998; Kemp and Manahan-Vaughan, 2004) and that de novo LTD was triggered by novel spatial learning in freely moving mice (Goh and Manahan-Vaughan, 2012). Discrepancies between these previous studies and the present work still need to be resolved but may be at least in part accounted for by species variations and different exploration protocols used in these studies.

Our pharmacological studies further demonstrate that this novel object exploration promoted LTD, like the previously reported hippocampal LTD produced by a stronger induction protocol in freely moving naive rats (Ge et al., 2010), is mechanistically mediated by activation of GluN2B-containing
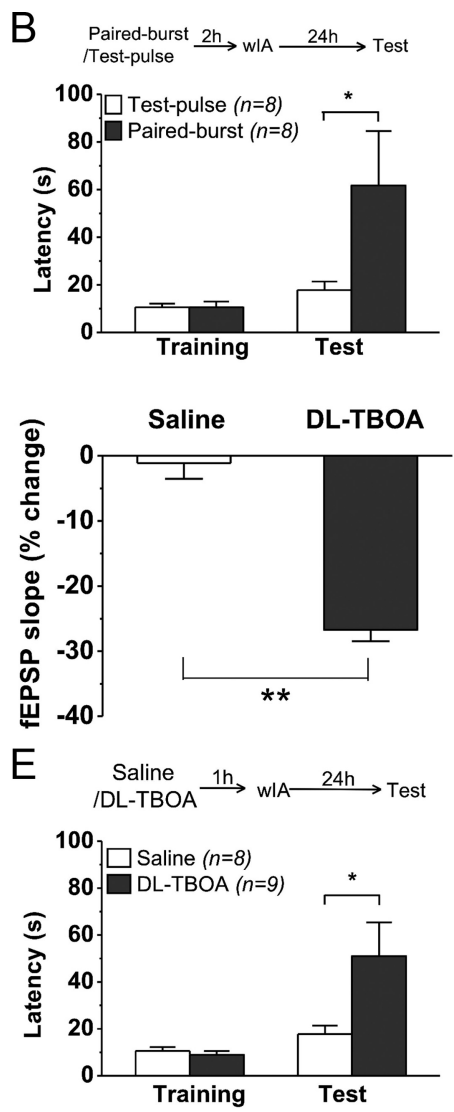

Saline $(n=4)$

- DL-TBOA $(n=5)$

Figure 6. Production of hippocampal LTD sufficiently promotes the conversion of wIA-induced STM into LTM. $\boldsymbol{A}, \boldsymbol{B}$, The plot of normalized fEPSPs $(\boldsymbol{A})$ and bar graph $(\boldsymbol{B})$ show that, $2 \mathrm{~h}$ before training, stimulation of Shaffer collateral inputs with the paired(paired burst; 200 pairs of 2 pulse bursts at 1 pair, with an interval of $2.5 \mathrm{~ms}$ between pulses and $10 \mathrm{~ms}$ $<0.001$ ) but also enabled the wIA training to form LTM tested $24 \mathrm{~h}$ after training $(B)\left(F_{(1,14)}=5.123 ; p=0.033\right)$. $C$, The plot (

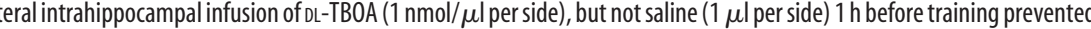
the wIA training to form LTM that could be revealed during the test performed $24 \mathrm{~h}$ after training $\left(F_{(1,15)}=5.030 ; p=0.040\right)$. ${ }^{*} p<0.05,{ }^{* *} p<0.01$. Error bars indicate SEM.

NMDARs during its induction and GluA2-dependent AMPAR endocytosis during its expression. This notion is further strengthened by the failure of Ro25-6981 (but not Tat-GluA2 3 ) to inhibit the LTD when given immediately after its induction. These results not only advance our understanding of the mechanisms underlying novel exploration facilitated hippocampal LTD but also validate the potential utility of the two structurally and mechanistically distinct LTD inhibitors in probing the role of this LTD in novelty exploration-related behaviors. Indeed, using these two inhibitors, we were able to provide direct evidence that the induction of LTD is responsible for the learning process of novelty acquisition. These results are in a good agreement with the recently reported role of LTD in object recognition (Griffiths et al., 2008; Seoane et al., 2009).

Novel environment is well known to enhance memory formation, but the underlying mechanisms remain largely unexplored. In the present study, we present several lines of evidence that NMDAR-dependent hippocampal CA1 LTD meets the major criteria, occurrence, necessity, and sufficiency (Martin et al., 2000; Collingridge et al., 2010) for its critical role in mediating memory enhancement by novel object exploration. First, as dis- 
cussed above, novel object exploration facilitates the induction of NMDAR-dependent hippocampal LTD. In addition, the fact that bilateral intrahippocampal application of LTD expression inhibitor Tat-GluA2 $2_{3 Y}$ (but not LTD induction inhibitor Ro25-6981) immediately after the novel object exploration and before wIA training remains effective in preventing the conversion of STM to LTM further demonstrates the occurrence of hippocampal CA1 LTD during the novel object exploration and before wIA training. It is interesting to note that such occurrence of LTD was not detected in our continuously recordings of fEPSPs during object exploration. One possible explanation may be due to the failure of fEPSP recordings used in the present study to detect object exploration-induced LTD under our recording conditions. For instance, object exploration, while reducing the threshold for inducting LTD in the vast majority of synapses at the CA1, may only produce LTD at a very small fraction of sparsely distributed CA1 synapses that are directly involved in the object recognition processes. The synaptic efficacy alteration at such a small number of sparsely distributed synapses may be obscured by vast unchanged synapses in the CA1 region under the current extracellularly recording, with a single electrode, of compound EPSPs generated from all the synapses stimulated by the stimulating electrode. Indeed, as previously reported, such behaviorally induced synaptic changes in a small fraction of sparsely distributed synapses may not be faithfully recorded with a single electrode but be better recorded with a more sophisticated multielectrode recording array (Whitlock et al., 2006).

Second, the two LTD inhibitors, although mechanistically and structurally different, similarly prevent the novel object exploration-promoted LTD and conversion of STM to LTM, indicating the necessity of the production of hippocampal LTD in this memory enhancement. Again, this notion was further supported by the different actions of Ro25-6981 and Tat-GluA2 $2_{3 Y}$ on the STM-to-LTM conversion when they were applied immediately after the novelty exploration and before wIA training. It should also be noted that neither Ro25-6981 nor Tat-GluA2 3 Y showed any notable effect on basal synaptic transmission, eLTP or LTP under our experimental conditions. Finally, using means other than novel object exploration, both electrical stimulation with paired burst protocol or pharmacological inhibition of glutamate transporter protocols, to induce hippocampal CA1 LTD, we were able to demonstrate the sufficiency of LTD production in promoting the conversion of wIA-induced STM into LTM. Together, our results strongly support the notion that NMDARdependent hippocampal LTD is not only present, and necessary, but also sufficient to mediate novelty exploration-induced memory enhancement.

However, how LTD production promotes the STM-to-LTM conversion remains unknown. In a recent study, we reported that production of hippocampal CA1 LTD is critically involved in mediating early consolidation of memory in a Morris water maze task (Ge et al., 2010). Thus, a plausible conjecture is that LTD production may increase the memory consolidation during and immediately after wIA training, thereby prolonging its memory traces in the neuronal circuit and consequently resulting in the conversion of STM into LTM. In addition, recent studies also proposed that novelty exploration may promote LTM formation through the mechanism of behavioral tagging and capture processes (Moncada and Viola, 2007; Ballarini et al., 2009). Behavioral tagging is derived from the synaptic tagging and capture that were originally postulated by Frey and Morris (1997). It was proposed that any learning behavior will produce a transient, protein synthesis-independent, and input-specific tag, but LTM forma- tion will additionally require the supply of new plasticity-related proteins. The STM induced by a weak training protocol could be extended to LTM, if there is a spatiotemporal overlapping between this weak training and a prior learning that produces a learning-related tag. However, the identity of such a putative learning tag has not been defined. The data from the current work reveal that novel object exploration dramatically facilitates hippocampal LTD, but not LTP, induction and plays a critical role in the conversion of STM into LTM. Notably, these effects of novel object exploration can be faithfully mimicked by pharmacological production of LTD with a glutamate uptake inhibitor. These results may support the view that hippocampal LTD, rather than the novel object exploration process per se, plays a vital role in the conversion of STM into LTM. Thus, LTD could potentially function as a behavioral tag, thereby promoting the conversion of STM to LTM.

It is interesting to note that the present work presents evidence suggesting a critical role of hippocampal LTD in two behaviorally and mechanistically distinct memory tasks: object recognition (a task that appears more to test the strength or "soundness" of the memory) and inhibitory avoidance (a procedure that is often used to test the incentive salience of memory). Moreover, we have also recently demonstrated a critical role of hippocampal LTD in promoting the consolidation of spatial learning in a water maze task (Ge et al., 2010). In addition, recent studies also suggest that induction of hippocampal CA1 LTD may also be involved in impairing memory retrieval if it is induced after the memory formation, but before the memory retrieval (Wong et al., 2007). Thus, hippocampal LTD may have diverse roles for LTD in many forms of learning and memory that are mediated by neuronal networks involving hippocampal CA1 synapses, and the timing of LTD induction may be critical in determining their specific effects on memory (Kemp and Manahan-Vaughan, 2007; Collingridge et al., 2010).

\section{References}

Ahmadian G, Ju W, Liu L, Wyszynski M, Lee SH, Dunah AW, Taghibiglou C, Wang Y, Lu J, Wong TP, Sheng M, Wang YT (2004) Tyrosine phosphorylation of GluR2 is required for insulin-stimulated AMPA receptor endocytosis and LTD. EMBO J 23:1040-1050.

Ballarini F, Moncada D, Martinez MC, Alen N, Viola H (2009) Behavioral tagging is a general mechanism of long-term memory formation. Proc Natl Acad Sci U S A 106:14599-14604.

Bliss TV, Collingridge GL (1993) A synaptic model of memory: long-term potentiation in the hippocampus. Nature 361:31-39.

Brebner K, Wong TP, Liu L, Liu Y, Campsall P, Gray S, Phelps L, Phillips AG, Wang YT (2005) Nucleus accumbens long-term depression and the expression of behavioral sensitization. Science 310:1340-1343.

Collingridge GL, Peineau S, Howland JG, Wang YT (2010) Long-term depression in the CNS. Nat Rev Neurosci 11:459-473.

Dong Z, Han H, Wang M, Xu L, Hao W, Cao J (2006) Morphine conditioned place preference depends on glucocorticoid receptors in both hippocampus and nucleus accumbens. Hippocampus 16:809-813.

Dong Z, Han H, Cao J, Zhang X, Xu L (2008) Coincident activity of converging pathways enables simultaneous long-term potentiation and longterm depression in hippocampal CA1 network in vivo. PLoS One 3:e2848.

Dudek SM, Bear MF (1992) Homosynaptic long-term depression in area CA1 of hippocampus and effects of $N$-methyl-D-aspartate receptor blockade. Proc Natl Acad Sci U S A 89:4363-4367.

Dudek SM, Bear MF (1993) Bidirectional long-term modification of synaptic effectiveness in the adult and immature hippocampus. J Neurosci 13:2910-2918.

File SE, Wardill AG (1975) Validity of head-dipping as a measure of exploration in a modified hole-board. Psychopharmacologia 44:53-59.

Fox CJ, Russell KI, Wang YT, Christie BR (2006) Contribution of NR2A and NR2B NMDA subunits to bidirectional synaptic plasticity in the hippocampus in vivo. Hippocampus 16:907-915. 
Frey U, Morris RG (1997) Synaptic tagging and long-term potentiation. Nature 385:533-536.

Ge Y, Dong Z, Bagot RC, Howland JG, Phillips AG, Wong TP, Wang YT (2010) Hippocampal long-term depression is required for the consolidation of spatial memory. Proc Natl Acad Sci U S A 107:16697-16702.

Goh JJ, Manahan-Vaughan D (2012) Spatial object recognition enables endogenous LTD that curtails LTP in the mouse hippocampus. Cereb Cortex. Advance online publication. Retrieved July 23, 2012. doi:10.1093/cercor/bhs089.

Griffiths S, Scott H, Glover C, Bienemann A, Ghorbel MT, Uney J, Brown MW, Warburton EC, Bashir ZI (2008) Expression of long-term depression underlies visual recognition memory. Neuron 58:186-194.

Honey RC, Watt A, Good M (1998) Hippocampal lesions disrupt an associative mismatch process. J Neurosci 18:2226-2230.

Izquierdo I, Medina JH (1997) Memory formation: the sequence of biochemical events in the hippocampus and its connection to activity in other brain structures. Neurobiol Learn Mem 68:285-316.

Jenkins TA, Amin E, Pearce JM, Brown MW, Aggleton JP (2004) Novel spatial arrangements of familiar visual stimuli promote activity in the rat hippocampal formation but not the parahippocampal cortices: a c-fos expression study. Neuroscience 124:43-52.

Kemp A, Manahan-Vaughan D (2004) Hippocampal long-term depression and long-term potentiation encode different aspects of novelty acquisition. Proc Natl Acad Sci U S A 101:8192-8197.

Kemp A, Manahan-Vaughan D (2007) Hippocampal long-term depression: master or minion in declarative memory processes? Trends Neurosci 30:111-118.

Lemon N, Manahan-Vaughan D (2006) Dopamine $\mathrm{D}_{1} / \mathrm{D}_{5}$ receptors gate the acquisition of novel information through hippocampal long-term potentiation and long-term depression. J Neurosci 26:7723-7729.

Leung LW (1979) Orthodromic activation of hippocampal CA1 region of the rat. Brain Res 176:49-63.

Liu L, Wong TP, Pozza MF, Lingenhoehl K, Wang Y, Sheng M, Auberson YP, Wang YT (2004) Role of NMDA receptor subtypes in governing the direction of hippocampal synaptic plasticity. Science 304:1021-1024.

Malenka RC, Nicoll RA (1993) NMDA-receptor-dependent synaptic plasticity: multiple forms and mechanisms. Trends Neurosci 16:521-527.

Malenka RC, Nicoll RA (1999) Long-term potentiation-a decade of progress? Science 285:1870-1874.

Manahan-Vaughan D (1998) Priming of group 2 metabotropic glutamate receptors facilitates induction of long-term depression in the dentate gyrus of freely moving rats. Neuropharmacology 37:1459-1464.

Manahan-Vaughan D, Braunewell KH (1999) Novelty acquisition is associated with induction of hippocampal long-term depression. Proc Natl Acad Sci U S A 96:8739-8744.

Martin SJ, Grimwood PD, Morris RG (2000) Synaptic plasticity and memory: an evaluation of the hypothesis. Annu Rev Neurosci 23:649-711.

Mellentin C, Abraham WC (2001) Priming stimulation of group II metabotropic glutamate receptors inhibits the subsequent induction of rat hippocampal long-term depression in vitro. Neurosci Lett 307:13-16.

Moncada D, Viola H (2007) Induction of long-term memory by exposure to novelty requires protein synthesis: evidence for a behavioral tagging. J Neurosci 27:7476-7481.

Morris RG, Garrud P, Rawlins JN, O'Keefe J (1982) Place navigation impaired in rats with hippocampal lesions. Nature 297:681-683.

Mulkey RM, Malenka RC (1992) Mechanisms underlying induction of ho- mosynaptic long-term depression in area CA1 of the hippocampus. Neuron 9:967-975.

O’Keefe JA, Nadel L (1978) The hippocampus as a cognitive map. Oxford: Clarendon.

Paxinos G, Watson C (2004) The rat brain in stereotaxic coordinates, 5th edition. Burlington, MA: Academic.

Platel A, Porsolt RD (1982) Habituation of exploratory activity in mice: a screening test for memory enhancing drugs. Psychopharmacology (Berl) $78: 346-352$.

Riedel G, Micheau J, Lam AG, Roloff EL, Martin SJ, Bridge H, de Hoz L, Poeschel B, McCulloch J, Morris RG (1999) Reversible neural inactivation reveals hippocampal participation in several memory processes. Nat Neurosci 2:898-905.

Seoane A, Massey PV, Keen H, Bashir ZI, Brown MW (2009) L-type voltagedependent calcium channel antagonists impair perirhinal long-term recognition memory and plasticity processes. J Neurosci 29:9534-9544.

Staubli U, Scafidi J (1997) Studies on long-term depression in area CA1 of the anesthetized and freely moving rat. J Neurosci 17:4820-4828.

Szapiro G, Izquierdo LA, Alonso M, Barros D, Paratcha G, Ardenghi P, Pereira P, Medina JH, Izquierdo I (2000) Participation of hippocampal metabotropic glutamate receptors, protein kinase A and mitogenactivated protein kinases in memory retrieval. Neuroscience 99:1-5.

Thiels E, Xie X, Yeckel MF, Barrionuevo G, Berger TW (1996) NMDA receptor-dependent LTD in different subfields of hippocampus in vivo and in vitro. Hippocampus 6:43-51.

Van den Oever MC, Goriounova NA, Li KW, Van der Schors RC, Binnekade R, Schoffelmeer AN, Mansvelder HD, Smit AB, Spijker S, De Vries TJ (2008) Prefrontal cortex AMPA receptor plasticity is crucial for cueinduced relapse to heroin-seeking. Nat Neurosci 11:1053-1058.

Wagner JJ, Alger BE (1995) GABAergic and developmental influences on homosynaptic LTD and depotentiation in rat hippocampus. J Neurosci 15:1577-1586.

Wang M, Yang Y, Dong Z, Cao J, Xu L (2006) NR2B-containing N-methylD-aspartate subtype glutamate receptors regulate the acute stress effect on hippocampal long-term potentiation/long-term depression in vivo. Neuroreport 17:1343-1346.

Whitlock JR, Heynen AJ, Shuler MG, Bear MF (2006) Learning induces long-term potentiation in the hippocampus. Science 313:1093-1097.

Wong TP, Howland JG, Robillard JM, Ge Y, Yu W, Titterness AK, Brebner K, Liu L, Weinberg J, Christie BR, Phillips AG, Wang YT (2007) Hippocampal long-term depression mediates acute stress-induced spatial memory retrieval impairment. Proc Natl Acad Sci U S A 104:11471-11476.

Xiong W, Wei H, Xiang X, Cao J, Dong Z, Wang Y, Xu T, Xu L (2004) The effect of acute stress on LTP and LTD induction in the hippocampal CA1 region of anesthetized rats at three different ages. Brain Res 1005:187-192.

Xu L, Anwyl R, Rowan MJ (1997) Behavioural stress facilitates the induction of long-term depression in the hippocampus. Nature 387:497-500.

Xu L, Anwyl R, Rowan MJ (1998) Spatial exploration induces a persistent reversal of long-term potentiation in rat hippocampus. Nature 394:891-894.

Yang CH, Huang CC, Hsu KS (2005) Behavioral stress enhances hippocampal CA1 long-term depression through the blockade of the glutamate uptake. J Neurosci 25:4288-4293.

Zhu XO, McCabe BJ, Aggleton JP, Brown MW (1997) Differential activation of the rat hippocampus and perirhinal cortex by novel visual stimuli and a novel environment. Neurosci Lett 229:141-143. 